Silva, J. A. (2015). Referentes conceptuales para la integración de un modelo de gobernanza del agua. Revista Lebret, 7. Bucaramanga, Colombia: Universidad Santo Tomás, pp. $21-35$. ISSN: 2145-5996.

\title{
Referentes conceptuales para la integración de un modelo de gobernanza del agua*
}

\author{
Conceptual references to the integration of \\ a water governance model
}

Jorge Alejandro Silva Rodríguez de San Miguel

\section{Resumen}

La mejora de la gobernanza del agua es fundamental para afrontar la inseguridad del recurso hídrico en los países en vías de desarrollo. Por ello, el objetivo de esta investigación documental es proponer un conjunto de referentes conceptuales para la integración de un modelo de gobernanza del agua, que coadyuve a diagnosticar los desafíos derivados de la gestión del agua. Se concluye que los referentes conceptuales deben integrar factores económicos, políticos, sociales y medio ambientales, y se sugiere profundizar en el tema para obtener resultados concretos de cómo integrar un modelo de gobernanza en un contexto determinado.

\section{Palabras clave}

Gobernanza del agua, modelos de gobernanza del agua, gestión del agua.

Códigos de Clasificación JEL: Q38, Q56.

\begin{abstract}
Improving water governance is essential to address water resource insecurity in developing countries. Therefore, the aim of this research paper is to propose a set of conceptual references for the integration of a water governance model that helps diagnose water management challenges. It is concluded that the conceptual references should integrate economic, political, social and environmental factors, and further research on the topic is suggested in order to obtain concrete results on how to integrate a water governance model in a given context.
\end{abstract}

\section{Keywords}

Water governance, water governance models, water management.

* $\quad$ Artículo derivado de la tesis doctoral "Diseño de un modelo para mejorar la gestión integral de agua potable en la delegación Iztapalapa de la ciudad de México". Apartes de este artículo fueron presentados como ponencia en el XX Congreso Internacional de Contaduría, Administración e Informática realizado del 7 al 9 de octubre en ciudad universitaria de México, D. F.

1 Doctor en Ciencias Administrativas. Docente en la Escuela Superior de Comercio y Administración Unidad Santo Tomás del Instituto Politécnico Nacional de México. Correo electrónico: j.a.silva@outlook.com 


\section{Introducción}

Investigadores en el tema del agua de la última década están de acuerdo con que la mejora de la gobernanza del agua es la clave para resolver la inseguridad del agua en los países en vías de desarrollo (Global Water Partnership, 2000; Rogers \& Hall, 2003; Kashyap, 2004; Gopalakrishnan, Tortajada, \& Biswas, 2005; Saleth \& Dinar 2005; Hoekstra \& Chapagain, 2007; Briscoe, 2009; Biswas \& Tortajada, 2010; OECD, 2011). El informe mundial de la visión del agua, por ejemplo, culpó a las malas instituciones, al Gobierno, a los incentivos y a las malas asignaciones de recursos como las causas fundamentales del problema (Cosgrove \& Rijsberman, 2000).

No hay consenso sobre la forma de abordar el estudio de la gobernanza del agua. Algunos investigadores como Saleth y Dinar (2005) emplean un enfoque comparativo con indicadores entre países sobre la base de 3 series de indicadores (ley de aguas, la política y la administración) y 20 sub-indicadores. Otros estudiosos como Biswas y Tortajada (2010) proponen un enfoque alternativo basado en estudios de casos independientes y objetivos de buenas prácticas de gobernanza del agua. En particular, su propuesta es estudiar los factores ambientales.

Los retos de la gobernanza del agua son más graves en los países en vías de desarrollo. Briscoe (2009) señala que los países pobres a menudo sufren el problema de la gobernanza del agua, más las frecuentes inundaciones y sequías, el rápido crecimiento de la demanda de agua, las pequeñas dotaciones de infraestructura de agua, las instituciones frágiles y la incertidumbre derivada del cambio climático. La revisión de la literatura muestra que hay poco consenso sobre el alcance y la definición de este fenómeno y que no hay un consenso de cómo integrar un modelo de gobernanza del agua en una demarcación determinada. Por ello, el objetivo de esta investigación es proponer un conjunto de referentes conceptuales para la integración de un modelo de esta naturaleza que permita diagnosticar los desafíos derivados de la gestión del agua.

\section{Método de investigación}

En esta investigación, de corte documental, se analizó la literatura de la gobernanza del agua en fuentes documentales diversas como libros, tesis de posgrado, documentos técnicos, sitios web y publicaciones científicas en diferentes bases de datos como JSTOR, EBSCO, Springer, Elsevier, entre otros. Asimismo, fueron revisados los principales conceptos y modelos de la gobernanza del agua bajo criterios de rigor científico. De cada modelo se precisaron sus enfoques disciplinarios, a partir de las teorías extraídas de la economía pública, la nueva economía institucional, la economía política y la administración pública. Estos referentes coadyuvaron a diagnosticar problemas asociados con la gobernanza del agua. 


\section{Conceptualización de la gobernanza del agua}

La Asociación Mundial del Agua define la gobernanza del agua como el rango de política, sistemas sociales, económicos y administrativos que existen para la gestión del agua, los recursos y la prestación de servicios de agua, en diversos niveles de la sociedad (Global Water Partnership, 2002). Esta definición, sin embargo, es problemática porque prácticamente la totalidad de la literatura sobre el agua recaería en aspectos de política, economía, finanzas y regulación. También sufre de un problema de especificación, porque los mecanismos para desarrollar y gestionar los recursos hídricos a menudo no están bien especificados, y por lo tanto sus implicaciones operativas para la investigación y la política de aguas no están claras. Por otro lado, el Fondo para la Gobernabilidad del Agua ofrece una definición más concreta: argumenta que la gobernanza del agua debe retomar principios de equidad y eficiencia en los recursos hídricos y la asignación de los servicios, la administración del agua basada en las cuencas, la necesidad del agua integrada, enfoques de gestión, y la necesidad de equilibrar el uso del agua entre las actividades socio-económicas y los ecosistemas. También pide la clarificación de las funciones del gobierno, de la sociedad y del sector privado, y sus responsabilidades con respecto a la propiedad y la gestión de recursos y servicios hídricos (UNDP Water Governance Facility, 2013). El concepto, no obstante, ofrece principios rectores e implicaciones políticas claras, pero no abunda en su utilidad.

Otros autores como Kashyap (2004) hacen referencia a la gobernanza del agua como la cualidad para desarrollar la capacidad de adaptación. Del mismo modo, Hirsch (2006) argumenta que la gobernanza debe entenderse como una manera para negociar de forma sostenible, equitativa y productiva. Tropp (2007), por el contrario, se refiere a la gobernanza del agua en términos de la evolución de las redes formales e informales, asociaciones, decisiones conjuntas que incluyen el diálogo y los resultados negociados como mecanismos de dirección de la gobernanza del agua. Mollinga (2008) emplea un enfoque de la sociología política y argumenta que la gobernanza del agua es un dominio político controvertido. Este enfoque puede tener valor teórico, pero no abunda sobre la política de los mecanismos que generan conflictos por el agua, las alternativas para resolverlos y sus implicaciones para la eficiencia, la equidad y la sostenibilidad. Pahl-Wostl, Gupta y Petry (2008) definen el término como el desarrollo y la aplicación de normas, principios, incentivos, herramientas informativas y de infraestructura para suscitar un cambio en el comportamiento de los actores a nivel mundial en el ámbito de la gobernanza del agua. Esta definición es similar a la de la Asociación Mundial del Agua y del Fondo para la Gobernabilidad del Agua, porque se enfoca en aspectos de política, economía, finanzas y regulación. Biswas y Tortajada (2010) argumentan que el término gobernanza del agua ha sido sustituido por los términos "gestión del agua sostenible" y "gestión integrada de recursos hídricos”. Finalmente, Wiek y Larson (2012) resumen algunas de las características clave de la gobernanza del agua como una perspectiva sistémica, un 
enfoque de gobernanza en los actores sociales, un discurso transparente y accesible en valores y metas, y una perspectiva amplia sobre la sostenibilidad del agua, siendo la definición más amplia de las analizadas.

Existe diversidad de opiniones sobre la definición del concepto gobernanza, mientras que en Europa el término refiere a las ideas de nueva gobernanza, asociadas al involucramiento de la sociedad en los procesos de gobierno; en Estados Unidos el término se asocia a las formas de coordinación (Mussetta, 2008). El concepto que concierne a México es el de la visión europea.

\section{Modelos de gobernanza}

\section{Gestión de instituciones formales de Saleth y Dinar (1999)}

Saleth y Dinar (1999) describieron indicadores para la evaluación de instituciones formales encargadas de la gestión del agua. Los indicadores tienen una orientación amplia que es aplicable a la evaluación del desempeño institucional; con estos se pretendió cuantificar a través de la efectividad de los elementos de las instituciones, las interrelaciones entre sus componentes y la relación entre las instituciones y el desempeño del sector del agua. Al analizar la eficacia de las instituciones, los autores descompusieron sus componentes constituyentes y se acercaron a los indicadores que tenían un efecto individual e interactivo con las instituciones sobre el desempeño institucional, así como a través del impacto socio-económico, político y medio ambiental.

El modelo considera los siguientes componentes de las instituciones: la ley, la política y la administración. Si se aplica este enfoque en el caso específico de las instituciones de gestión del agua, el modelo se definiría en términos de la ley, la política del agua y la administración del agua. Los países que han adoptado el modelo son Australia, Brasil, Chile, China, India, Israel, Sudáfrica, España, Sri Lanka y Estados Unidos (Saleth \& Dinar, 1999). Es un modelo orientado a la efectividad y se inclina hacia la política.

\section{Modelo de gobernanza de Fung y Wright (2003)}

En el modelo de gobernanza de Fung y Wright, el enfoque llega para presentarse como una forma no jerárquica de gobierno, caracterizada por la cooperación con actores no estatales en el interior de redes de decisión mixtas entre lo público y lo privado, los procesos gubernamentales aluden a una concepción de buen gobierno en el que se destaca a los actores no gubernamentales y se les incluye en los procesos de toma de decisión para la producción de políticas públicas y programas de gobierno. Se supone que un Estado que promueve la participación en este sentido es más flexible y capaz de descentralizar funciones, transferir responsabilidades y ampliar el universo 
de actores participantes, claro que sin que ello implique el abandono de instrumentos de control y supervisión. Al mismo tiempo, estas nuevas modalidades de gestión de gobierno pretenden potenciar los derechos de los ciudadanos. La gobernanza se asocia al buen gobierno, porque además de las preocupaciones sistémicas más fuertes del modelo de la gobernanza (eficacia y eficiencia en la resolución de problemas), incorpora una dimensión normativa: el gobierno debe ser a la vez promotor de la profundización de la democracia a través de mejores sistemas de representación de intereses (Mussetta, 2008).

El modelo ha sido aplicado en México como una estrategia progresiva de reforma institucional, confronta los valores de la participación, la delegación de poder y la deliberación, con los límites concretos de la prudencia y la viabilidad, lo cual profundiza la concepción teórica y empírica de la práctica democrática. Aquí, un rediseño institucional con participación de la sociedad civil surge como respuesta a determinadas fallas en ciertas organizaciones administrativas y regulatorias específicas (Mussetta, 2008).

\section{Modelo de gobernanza del agua de la Unesco (2006)}

Este modelo teórico de la Unesco se enfoca en temas gubernamentales, cuya finalidad es la formulación de políticas y de su ejecución por parte del Estado (Unesco, 2006). El modelo se integra por las dimensiones social, económica, de la capacitación política y de la sostenibilidad medioambiental.

La dimensión social hace referencia a un uso equitativo de los recursos hídricos. El agua no solo se distribuye de manera heterogénea en el tiempo y el espacio; sino también entre los diferentes estratos socioeconómicos de una sociedad, tanto en los asentamientos rurales como en los urbanos. La dimensión económica describe el uso eficiente de los recursos hídricos y el rol del agua en el crecimiento económico global. Las perspectivas de una fuerte reducción de la pobreza y de crecimiento económico siguen dependiendo, en gran medida, del agua y de otros recursos naturales. Los ingresos per cápita y la calidad de la gobernabilidad están reducidamente interrelacionados de manera positiva en diversos países, debido a esto una mejor gobernabilidad ejerce un gran efecto en los ingresos per cápita. La dimensión de la capacitación política toma en cuenta a las partes concernidas y a los ciudadanos, en igualdad de oportunidades democráticas, para influir y controlar los procesos políticos y sus resultados. Los ciudadanos marginados tanto a nivel nacional como internacional pueden ser los pueblos indígenas, las mujeres, los habitantes de barrios marginales, entre otros. Otorgar poder a las mujeres, al igual que a otros grupos marginales es decisivo para conseguir una gestión equilibrada y eficaz. La dimensión de sostenibilidad medioambiental explica que una mejor gobernabilidad permite un uso sostenible del agua y la integridad del ecosistema. Un flujo suficiente de recursos hídricos de calidad es fundamental para conservar las 
funciones, servicios del ecosistema y sustentar los acuíferos, los humedales y otros hábitats naturales (Unesco, 2006).

Por otro lado, la gobernabilidad es uno de los temas sobre el agua de mayor relevancia en el mundo (Unesco, 2006), y su modelo es aplicable a cualquier país en el que los servicios de agua sean total o parcialmente propiedad pública. Algunos ejemplos de países que tienen la propiedad pública de los servicios de agua son Austria, Canadá, Dinamarca, Finlandia, Grecia, Irlanda, Italia, Japón, Corea, Luxemburgo, Polonia, Suecia, Suiza y Turquía, etc. (Organisation for Economic Cooperation and Development, 2009).

\section{Modelo leviatán hidráulico de Mussetta (2008)}

En este modelo explicado por Mussetta (2008) se hace énfasis en un Estado centralizado, fuerte en términos de presencia estatal. Un Estado que planifica y desarrolla, que construye grandes obras de infraestructura hidráulica (diques, represas, sistemas de riego) y en esto se mide el potencial de su fuerza. Es a su vez un Estado que organiza a los demás actores de la economía y de la sociedad, y nunca delega la gerencia de este puesto. Cronológicamente, al leviatán hidráulico se le puede ubicar a partir de la década del sesenta en países de Latinoamérica como México, donde agua era sinónimo de desarrollo y no se concebía públicamente la posibilidad de que otro actor distinto del Estado interfiriera en sus asuntos, la Federación era la que decidía y tenía legítima autoridad sobre el recurso agua. En este modelo el Estado ocupa un papel de transformador de la sociedad en nombre del bienestar público general pero con rigidez.

\section{Modelo de línea dura y blanda de Guhl (2008)}

El modelo teórico descrito por Guhl (2008), en la línea dura, se enfoca en aumentar la oferta de agua y hacerla más estable en el tiempo y en el espacio, mediante la construcción de obras de infraestructura que permiten contar con una oferta mayor y más estable de agua a lo largo del año, o una disponibilidad en zonas que presentan déficit. En esta línea no se considera el tema de la producción natural del agua mediante la conservación y restauración de los ecosistemas productores y protectores. Además, la participación de los usuarios en la planificación y gestión del recurso es escasa. La Línea Dura ha sido la más utilizada por los gobiernos y por la banca multilateral de desarrollo, por su implicación técnica - económica en cuanto a la construcción de grandes obras, pero es insostenible en el tiempo y produce impactos ambientales muy fuertes, que son muchas veces irreversibles, sobre los ecosistemas y sobre la sociedad.

En la línea blanda la gestión se basa en la búsqueda de la sostenibilidad de disponibilidad, a partir de la racionalización de los usos y el consumo y se caracteriza por tener impactos ambientales menores que los que implica la línea dura. Su objetivo 
fundamental es la conservación de la oferta de agua y su uso eficiente, da consideración integral al ciclo hidrológico con base en cambios de actitudes y comportamientos de los usuarios con respecto a la valoración del agua como un recurso finito y la consecuente limitación de su disponibilidad y el desarrollo y empleo de tecnologías más eficientes (Guhl, 2008). Con esta perspectiva, la planeación de la gestión se realiza siguiendo el ciclo hidrológico, es decir, con una visión integral del recurso desde su generación hasta su tratamiento final y reúso.

\section{Modelo de gestión integrada del agua de Guhl (2008)}

El modelo teórico de la gestión integrada del agua explicado por Guhl (2008) pretende promover el manejo y el desarrollo coordinado del agua, la tierra y los recursos relacionados con el fin de maximizar el bienestar social y económico resultante de manera equitativa; sin comprometer la sustentabilidad de los ecosistemas vitales. La importancia del concepto es que maneja la variable ambiental, social y económica (Mussetta, 2008).

La gestión integrada del agua es un modelo teórico que tiene tres principios centrales que están de una u otra manera en todas sus definiciones: equidad, eficacia y sostenibilidad. Además, implica una visión de la relación sociedad-naturaleza, basada en un marco conceptual orientado a gestionar la búsqueda de la sostenibilidad y el desarrollo de los recursos hídricos. Lograrla supone que la población cambie las prácticas insostenibles de consumo y de uso, adquiera una visión de conjunto sobre los efectos de las acciones individuales y se dé cuenta de que los impactos que causa cada individuo, aparentemente insignificantes, se agregan a los de los demás amplificándose por millones (Guhl, 2008).

\section{Modelo de gestión del agua por intermunicipalidad de Ventura (2010)}

Deacuerdocon Ventura(2010), el modelodegestión del agua porintermunicipalidad ha surgido como consecuencia de la "descentralización" del servicio de agua potable y saneamiento. Además, busca crear diversos vínculos entre organizaciones gubernamentales que quieren influir en el diseño y la implementación de las políticas públicas (Rhodes, 2000). Con el modelo se pretende que exista una mejor gestión por medio de un organismo que integre varios municipios y que comparta responsabilidades con otro organismo que gestiona otros municipios. Se llevó a cabo un estudio en las regiones de San Luis Potosí y Pachuca de México, en el que se consideraron las categorías siguientes: a) la autonomía del organismo intermunicipal; b) la estructuración de relaciones con usuarios e instancias gubernamentales, y c) los obstáculos en el sistema intermunicipal. La investigación es novedosa por la incorporación de redes entre municipios, no obstante, es relativamente nueva en México (Ventura, 2010). 


\section{Enfoques disciplinarios de la gobernanza del agua}

De forma complementaria a los modelos, existen los siguientes enfoques disciplinarios de la gobernanza del agua planteados por Araral y Wang (2013).

\section{Economía pública}

La Economía pública se ocupa principalmente de cómo las políticas públicas afectan el bienestar social a través de un diagnóstico de un mercado. Se han catalogado diversas implicaciones de las fallas del mercado en el sector del agua. Por ejemplo, cuando las aguas avanzan en el sentido de la corriente y al revés en las cuencas hidrográficas, lo cual puede ocasionar problemas ambientales, económicos y sociales. Los costos políticos y administrativos de transacción en las grandes cuencas fluviales son altos y las capacidades de los gobiernos de los países en desarrollo siguen siendo débiles. Además, los activos sin precio y los mercados perdidos, o algún aspecto de los servicios de agua como el control de inundaciones no tienen un precio que pueda conducir a su provisión y producción sub-óptima. Los mercados perdidos de agua han proporcionado la base teórica de propuestas para facilitar o simular el funcionamiento de los mercados de agua a través de la cesión o la subasta de derechos de agua. Los mercados del agua se encuentran en funcionamiento en el suroeste de Estados Unidos y Australia. Sin embargo, en algunos países en vías de desarrollo los costos de transacción prohibitivos y los débiles marcos institucionales hacen que el comercio del agua sea desafiante. De igual forma, el control de inundaciones y la protección de la calidad del agua a menudo pueden ser bienes públicos; mientras que la mayoría de las inversiones al agua implican obtener economías de escala que crean monopolios naturales. Las economías de escala han proporcionado la base teórica para el continuo papel central del gobierno para realizar inversiones en el sector del agua con implicaciones en el diseño de los mecanismos de asignación de riesgo para las asociaciones público-privadas del agua. Por último, la incertidumbre sobre el agua hace que sea difícil para los mercados establecer los precios correctamente (Araral \& Wang, 2013).

Los gobiernos de todo el mundo regulan el precio del agua, la calidad y el nivel de servicio, al menos el 85\% de las utilidades del agua en todo el mundo están bajo el control de los gobiernos, pero también se presenta el caso de que la mayoría de estas utilidades son pobremente utilizadas. Muchos estudios sugieren brindar autonomía a los gobiernos para eficientar las utilidades del agua. Por otro lado, el comercio del agua se ha propuesto como una solución clave para el problema de la escasez de agua. Sin embargo, los costos prohibitivos del comercio del agua y los derechos poco claros han llevado a los gobiernos a tomar un papel más activo en la facilitación de la aparición de agua cuasi-mercados, a través de la infraestructura legal y la asignación de derechos de agua que de otra forma fallarían (Araral \& Wang, 2013). 
Un concepto relacionado con el comercio directo de agua es la noción de aguas virtuales. Su implicación central es que los países con escasez de agua podrían importar el uso intensivo del agua en productos agrícolas de países con abundante agua, durante el uso de sus recursos hídricos nacionales limitados para actividades de mayor valor. Una idea similar es la noción de huella hídrica, mientras la cuenca de un río puede ser la unidad de análisis para la planificación y los arreglos institucionales, esto no siempre puede ser suficiente para hacer frente a problemas contemporáneos sobre el agua. Esto se debe a que muchos tienen una dimensión global, lo que exige un enfoque de gobernanza que comprenda la coordinación y los arreglos institucionales en un nivel superior a la de la cuenca de un río (Abu-Sharar, Al-Karablieh, \& Haddadin, 2012; Araral \& Wang, 2013).

\section{Nueva economía institucional}

En este apartado se revisan aspectos de la teoría de los contratos incompletos y los costos de transacción. La teoría de los contratos incompletos tiene varias implicaciones importantes para la gobernanza del agua. Por ejemplo, la teoría sugiere que los resultados ineficientes en el suministro de agua pueden ser entendidos como una función de contratos incompletos, no especificados o renegociados. Del mismo modo, la teoría de la transacción del costo sugiere que la especificación completa de los derechos de agua y el cumplimiento de los contratos es problemático y el oportunismo por partes contratantes es inherente. Además, se puede presentar que no haya óptima estructura del agua del gobierno. En la práctica, la teoría de los contratos incompletos y los costos de transacción se puede utilizar para ayudar a diagnosticar y posiblemente resolver una variedad de problemas de gobernanza del agua importantes. Por ejemplo, las teorías pueden explicar el fracaso de la privatización de servicios del agua, porque las empresas se mantienen controladas por los gobiernos a pesar de sus deficiencias, porque los contratos de concesión de agua se renegocian o son devueltos al gobierno o porque existen dificultades para crear un mercado para el comercio de agua en países en vías de desarrollo, donde los derechos de agua no están claros, así como los retos contractuales de la creación de mercados de agua virtual utilizando huellas hídricas (Araral \& Wang, 2013).

\section{Economía política}

Este enfoque se ocupa principalmente de la interacción entre la política y los aspectos distributivos de la reforma del agua. La mejora de los derechos del agua, la fijación de precios de costo marginal y la privatización de los servicios públicos, entre otros, son estrategias débiles acerca de las dimensiones políticas del problema que explica por qué hay muy pocas reformas de gobernanza del agua exitosas. Tal vez el estudio empíricamente y teóricamente más significativo sobre la economía política de la reforma del agua urbana es el caso de seis reformas urbanas de agua en África y América Latina, en el cual se examinó la política, la economía y la 
institucionalidad (reglamentario, judicial, legal y político) como determinantes de las reformas de suministro de agua urbana exitosas y fallidas (Araral \& Wang, 2013). Clarke y Xu (2004) igualmente emplean un análisis de la economía política para explicar los determinantes de éxito en empresas privadas de agua en seis estudios de caso de África y América Latina. La reforma del sector del agua debe ser políticamente deseable, que es más probable cuando el público operador está funcionando mal y cuando los partidarios del gobierno se benefician de la reforma por lo general a través de la mejora de la calidad, la expansión del sistema o de la reducción de subvenciones. Además, se argumenta que la reforma tiene que ser políticamente factible y sugieren que si el Gobierno depende en gran medida de los grupos que van a perder de la reforma a través de aumentos de precios o la pérdida de empleos, la reforma será poco probable. Konca (2005), por otro lado, ofrece un tratamiento de la política transnacional de la gobernanza del agua utilizando la teoría de las relaciones internacionales. Argumenta que las amenazas al mundo de bienes comunes del agua han resistido el establecimiento de acuerdos globales de agua o interestatales mediante la negociación intergubernamental. Sin embargo, mientras la diplomacia del agua interestatal ha flaqueado, organizaciones menos formales han surgido para ayudar a mejorar la gobernanza del agua a nivel local y global (Araral \& Wang, 2013).

\section{Administración pública}

La administración pública se ocupa de la cuestión de por qué la mayoría de los servicios de agua y las organizaciones de riego en el desarrollo de los países son ineficientes. Su enfoque operativo está en la organización y gestión determinantes del desempeño de las burocracias acuáticas como la financiera y la de la autonomía de personal, las prácticas de gestión de recursos humanos, tales como la compensación, desarrollo de liderazgo, formación, estructura de incentivos, y los problemas generalmente asociados con fallas del gobierno. Los problemas se deben a que los directores no tienen exactamente los mismos intereses que sus gestores y porque es costoso para los directores supervisar a sus gestores. Los gestores tienen más información sobre sus actividades que sus directores, lo que permite que persigan sus propios intereses, en cierta medida. El director entonces se enfrenta a la tarea de crear disposiciones organizativas que minimicen la suma de los costos de la conducta indeseable de los gestores y de la actividad realizada para controlarlo (Araral \& Wang, 2013). Rogers (2002) cita los siguientes ejemplos de fracaso del gobierno en el sector del agua: la corrupción, la regulación de precios, los subsidios a los usuarios de recursos y contaminadores, impuestos, incentivos y créditos inapropiados, exceso de regulación o baja regulación, obstáculos burocráticos o inercia, regímenes normativos 
en conflicto y la información imperfecta. Por otra parte, el fracaso del gobierno también está estrechamente asociado con un sinnúmero de problemas: captura por grupos especiales de interés (operadores de agua, industrias, agricultores, etc.), pocos incentivos por los burócratas de la eficiencia operativa, la reflexión imprecisa de las preferencias del consumidor (debido a características de monopolio de abastecimiento urbano de agua), la incapacidad del gobierno para controlar y regular el uso sostenible de los recursos hídricos (debido a problemas de fragmentación y de información) y la independencia y la imparcialidad de los organismos reguladores (debido a las características políticas de agua).

\section{Discusión y propuesta del modelo de gobernanza del agua}

El término gobernanza fue definido por diversos autores (Global Water Partnership, 2002; Kashyap, 2004; Hirsch, 2006; Tropp, 2007; Mollinga, 2008; PahlWostl, Gupta, \& Petry, 2008; Biswas \& Tortajada, 2010), pero destaca el concepto de Wiek y Larson (2012) por concebir el término como un enfoque sistémico en actores sociales por medio de un discurso transparente y accesible en valores y metas, y una perspectiva amplia sobre la sostenibilidad del agua.

El modelo de gobernanza de Fung y Wright de 2003 (Mussetta, 2008) se caracteriza por la cooperación del Estado con actores no estatales en el interior de redes de decisión mixtas entre lo público y lo privado. No obstante, al delegar funciones el Estado, se puede perder la coordinación y los intereses principales por parte de los organismos subordinados. El modelo de gobernanza de la Unesco (2006) se centra en el aspecto político del recurso hídrico. El modelo leviatán hidráulico (Mussetta, 2008) se caracteriza por el control del Estado centralizado en la gestión que propicia una toma de decisiones lenta. El modelo de gestión del agua por intermunicipalidad (Ventura, 2010) integra a diversos organismos de distintos municipios y busca la agilización de las funciones de cada uno mediante el apoyo mutuo. Sin embargo, puede existir divergencia entre intereses de cada municipio. Los modelos de línea dura y blanda (Guhl, 2008) se enfocan en la oferta del agua que ocasiona un uso indiscriminado del recurso y en el uso racional de este basado en la demanda, respectivamente. Los enfoques disciplinarios complementan los referentes conceptuales de los modelos: la economía pública se enfoca en la afectación del mercado hídrico por políticas públicas, la nueva economía institucional se centra en contratos incompletos y costos de transacción entre organizaciones gestoras de agua, la economía política en el análisis de las reformas hídricas y la administración pública en la eficiencia en los servicios de agua (Araral \& Wang, 2013) (tabla 1). 
Tabla 1. Referentes conceptuales para la integración de un modelo de gobernanza del agua

\begin{tabular}{l|l}
\hline Modelo/ Enfoque disciplinario & Referente conceptual \\
\hline $\begin{array}{l}\text { Gestión de instituciones formales } \\
\text { (Saleth \& Dinar, 1999) }\end{array}$ & $\begin{array}{l}\text { Autonomía del organismo intermunicipal, } \\
\text { estructuración de relaciones y obstáculos en sistema } \\
\text { intermunicipal. }\end{array}$ \\
\hline $\begin{array}{l}\text { Gobernanza de Fung y Wright en 2003 } \\
\text { (Mussetta, 2008) }\end{array}$ & $\begin{array}{l}\text { Cooperación con actores no estatales, eficacia y } \\
\text { eficiencia en la resolución de problemas y normatividad } \\
\text { adecuada. }\end{array}$ \\
\hline Gobernanza del agua ( Unesco, 2006) & $\begin{array}{l}\text { Social, económica, capacitación, política y } \\
\text { sostenibilidad medioambiental. }\end{array}$ \\
\hline Leviatán hidráulico (Mussetta, 2008) & Centralización de la toma de decisiones \\
\hline Línea dura y blanda (Guhl, 2008) & Planeación de la oferta y demanda del agua \\
\hline Gestión integrada (Guhl, 2008) & Equidad, eficacia y sostenibilidad \\
\hline $\begin{array}{l}\text { Gestión del agua por intermunicipalidad } \\
\text { (Ventura, 2010) }\end{array}$ & Ley, política y administración \\
\hline Economía Pública (Araral \& Wang, 2013) & Afectación del mercado por políticas públicas \\
\hline $\begin{array}{l}\text { Nueva Economía Institucional } \\
\text { (Araral \& Wang, 2013) }\end{array}$ & Contratos incompletos y costos de transacción \\
\hline Economía Política (Araral \& Wang, 2013) & Política y reforma del agua \\
\hline Administración Pública (Araral \& Wang, 2013) & Eficiencia en los servicios de agua \\
\hline
\end{tabular}

Fuente: Autor con base en los modelos planteados por los autores enunciados en la tabla.

Para la integración de un modelo de gobernanza del agua, se deben tomar en cuenta los referentes más importantes, localizados en la literatura sobre la gobernanza del agua, para tener más elementos que fortalezcan el fenómeno, y no solo retomar un modelo en particular (tabla 1). Los referentes integran factores económicos, sociales, políticos y medio ambientales, y no aluden a su aplicación en un contexto específico, eso depende de los escenarios en los que se sitúe la gobernanza, considerando sus limitaciones. Los referentes del modelo de gobernanza de Fung y Wright de 2003 (Mussetta, 2008) dejan libertad para elegir entre una gobernanza público, privada o mixta. En el caso de la Unesco (2006), los factores sociales, económicos, políticos y medioambientales tienen lugar en la gobernanza. Para el caso de los referentes del modelo leviatán hidráulico (Mussetta, 2008) la centralización depende de la forma de gobierno del Estado en el que se aplique, ya sea centralizado o se puede optar por las redes intermunicipales (Ventura, 2010). También, es menester evaluar si el Estado opta por un enfoque de gobernanza en la oferta o demanda del agua (Guhl, 2008). De los enfoques disciplinarios, la economía pública, la nueva economía institucional, la economía política y la administración son variables que se tienen en cuenta (Araral \& Wang, 2013). Con los referentes se pretende tener una gobernanza del agua completa que contemple la gestión del agua desde su captación por cuencas hasta su distribución. 


\section{Conclusiones}

La mayoría de las investigaciones coinciden en que la mejora de la gobernanza del agua es la clave para hacer frente a la inseguridad del agua en los países en vías de desarrollo. Además, en la literatura sobre la gobernanza del agua hay poco consenso sobre el alcance y la definición de este término y no proporciona un análisis sólido de los problemas de incentivos que sostienen el núcleo de la gobernanza del agua y cómo se debe abordar su estudio. No obstante, la definición del concepto gobernanza que concierne a México es el de la visión europea que involucra a la sociedad en los procesos de gobierno.

Sobre la base de la revisión de la literatura, se abogó por una investigación interdisciplinaria de gobernanza del agua que integra los principales modelos localizados en la literatura y los enfoques interdisciplinarios de actualidad. Cada uno de los referentes se enfoca en un aspecto de la gobernanza, por lo que no hay un consenso de cómo integrar un modelo de gobernanza del agua.

Los referentes del modelo se retomaron de las investigaciones de Araral y Wang (2013), Guhl (2008), Mussetta (2008), Saleth y Dinar (1999), Unesco (2006) y Ventura (2010). En ellos se analizan factores necesarios para integrar la gobernanza del agua: políticos, económicos, sociales y medio ambientales.

Por último, se sugiere realizar mayor investigación sobre la gobernanza del agua en diferentes contextos para tener una visión integral de este fenómeno que pueda servir como impulso para integrar un modelo de esta naturaleza acorde con los desafíos presentes derivados de la escasez del agua, ya que los referentes que se plantean son teóricos, generales y dejan a libre albedrío de un gestor el cómo utilizarlos en un contexto determinado.

\section{Referencias}

Abu-Sharar, T. M., Al-Karablieh, E. K., \& Haddadin, M. J. (2012). Role of Virtual Water in Optimizing Water Resources Management in Jordan. Water Resources Management, 26(14), 3977-3993.

Araral, E., \& Wang, Y. (2013). Water Governance 2.0: A Review and Second Generation Research Agenda. Water Resources Management, 27(11), 3945-3957.

Biswas, A. K., \& Tortajada, C. (2010). Future water governance: problems and perspectives. International Journal of Water Resources Development, 26(2), 129139.

Briscoe, J. (2009). Water security: why it matters and what to do about it. Innovations, $4(3), 3-28$. 
Clarke, G., \& Xu, C. (2004). Privatization, competition, and corruption: how characteristics of bribe takers and payers affect bribe payments to utilities. Journal of Public Economics, 88, 2067-2097.

Cosgrove, W. J., \& Rijsberman, F. R. (2000). World water vision: making water everybody's business. London: Earthscan.

Global Water Partnership. (2000). Towards water security: a framework for action. Stockholm: Global Water Partnership.

Global Water Partnership. (2002). Effective water governance. Stockholm: Global Water Partnership.

Gopalakrishnan, C., Tortajada, C., \& Biswas, A. K. (2005). Water institutions: policies, performance and prospects. Berlin: Springer.

Guhl, E. (2008). Hacia una gestión integrada en la región Andina (Documentotécnico). Bogotá: Comunidad Andina.

Hirsch, P. (2006). Water governance reform and catchment management in the Mekong region. The Journal of Environment Development, 15(2), 184-201. doi: $10.1177 / 1070496506288221$

Hoekstra, A. Y., \& Chapagain, A. K. (2007). Water footprints of nations: water use by people as a function of their consumption pattern. Water Resources Management, 21(1), 35-48. doi:10.1007/s11269-006-9039-x.

Kashyap, A. (2004). Water governance: learning by developing adaptive capacity to incorporate climate variability and change. Water Science and Technology, 49(7), 141-146.

Konca, K. (2005). Governing water: contentious transnational politics and global institution building. Boston: MIT Press.

Mollinga, P. P. (2008). Water, politics and development: framing a political sociology of water resource management. Water Alternatives, 1(1), 7-23.

Mussetta, P. (2008). Participación y gobernanza. El modelo de gobierno del agua en México. Red de Revistas Cientificas de América Latina, el Caribe, España y Portugal, 12(25), 66-84.

OECD (2011). Water governance in OECD countries: a multi-level approach. doi:10.1787/9789264119284-en

Organization for Economic Co-operation and Development. (2009). Managing Water for All. Paris: OCDE.

Pahl-Wostl, C., Gupta, J., \& Petry, D. (2008). Governance and the global water system: a theoretical exploration. Global Governance, 14(4), 419-435. 
Rhodes, R. A. W. (2000). Governance and Public Administration. En Pierre, J. (Ed.). Debating Governance: Authority, Steering, and Democracy (pp. 54-90). Oxford: University Press.

Rogers, P. (2002). Water governance in Latin America and the Caribbean. Washington, D.C: Inter-American Development Bank.

Rogers, P., \& Hall, A. W (2003). Effective water governance. (Report No. 7). Stockholm: Global Water Partnership.

Saleth, R. M., \& Dinar, A. (1999). Evaluating Water Institutions and Water Sector Performance. World Bank Technical Paper No. 447. Washington, D.C.: The World Bank.

Saleth, R. M., \& Dinar, A. (2005). The institutional economics of water: a crosscountry analysis of institutions and performance. Cheltenham y Northampton: The World Bank.

Tropp, H. (2007). Water governance: trends and needs for new capacity development. Water Policy, 9(2), 19-30. doi:10.2166/wp.2007.137

UNDP Water Governance Facility. (2013). What is water governance? Recuperado de http://www.watergovernance.org/whatiswatergovernance

Unesco. (2006). El agua, una responsabilidad compartida. Segundo informe de las Naciones Unidas sobre el desarrollo de los recursos hídricos en el mundo. Recuperado de http://hispagua.cedex.es/sites/default/files/2\%C2\% BAInforme retos_gobernabilidad.pdf

Ventura, M. (2010). La gestión intermunicipal del agua en México. VertigO - la revue électronique en sciences de l'environnement, 7. doi:10.4000/vertigo.9692

Wiek, A., \& Larson, K. L. (2012). Water, people, and sustainability - a systems framework for Analyzing and assessing water governance regimes. Water Resources Management 26(11), 3153-3171. doi:10.1007/s11269-012-0065-6 